\title{
Classification of ECoG Motor Image using Fusion Technique
}

\author{
Aswinseshadri K \\ HCL Technologies Ltd \\ Chennai
}

\author{
Thulasi Bai V \\ Prathyusha Institute of Technology and \\ Management \\ Chennai
}

\begin{abstract}
Brain-Computer Interfaces (BCIs) ensure non-muscular communication between a user and external device by using brain activity. Currently, BCIs were applied in the medical field to increase quality of life of patients suffering from neuromuscular disorders. Most BCI systems use scalp recorded electroencephalographic activity, while Electrocorticography (ECoG) is a minimally-invasive alternative to Electroencephalogram (EEG), which ensures higher and superior signal characteristics enabling rapid user training and quicker communication. This paper presents a BCI system; ECoG signals are pre-processed and features are extracted from using Wavelet Packet Tree and Common Spatial Pattern. The extracted features are fused using Median Absolute Deviation (MAD) to improve the discrimination power of the feature vector. BCI Competition III, Data Set I having ECoG recordings motor imagery is used in investigation to evaluate the presented methodology.
\end{abstract}

\section{General Terms}

Pattern Recognition

\section{Keywords}

Brain-computer interface (BCI), Electrocorticography (ECoG), Wavelet Packet Tree, Common Spatial Pattern, Motor Imagery

\section{INTRODUCTION}

Essentially, BCI monitors brain activity through brain imaging technology, detecting characteristic brain pattern alterations through which user controls or communicates through devices. Messages and commands in BCI are expressed via brain generated electro-physiological signals and not through muscle contractions as in conventional communication [1]. Most BCIs are based on Electroencephalogram (EEG) as it is a non-invasive method to record electrical fields directly produced by neuronal synaptic activity [2]. Electrocorticogram (ECoG) is a minimallyinvasive EEG alternative to $\mathrm{BCI}$ providing superior signal characteristics which ensure rapid user training and quicker communication. Studies reveal that brain regions like auditory cortex can control a BCI system with methods similar to those training a brain's motor regions. This is important for users with neurological disease, head trauma, or conditions which prevent sensorimotor cortex use for BCI control [3].

Measurements from cortical surface with subdural electrodes are called Electrocorticogram (ECoG) and using a depth probe is called electrogram. ECoG is an invasive recording technique where electrodes, for example an 8 -by- 8 grid, are surgically placed beneath the skull. Strips with smaller numbers of electrodes are also inserted into deeper brain regions. ECoG, unlike invasive microelectrode recording techniques, measures large cell population's generated activity. So, ECoG measurements are comparable to extra cranial EEG, but electrode's proximity to cortex and lack of intervening tissue ensures higher signal-to-noise ratio, better response at higher frequencies, and drastic reduction in spatial blurring between nearby electrode signals and artefacts contamination. ECoG brain signals are advantageous when used with BCI systems compared to EEGs which include increased spatial resolution, signal bandwidth and larger signal amplitude. So, independent signals are differentiated over a frequencies range - on neighbouring electrodes - using procedures with motor and sensory imagery [4, 5]. Traditionally, motor imagery was resorted to as it was felt to be more accessible and reliable EEG signal. But, ECoG's advantages ensures that subjects learn multiple modalities use - including motor/sensory imagery - to control BCI applications thereby enabling individuals with damaged motor cortex due to stroke or neurological diseases to benefit from BCI systems.

ECoG-based BCI control methodology is based on use of sensorimotor rhythms. Primary sensorimotor rhythms present are $\mu(8-12 \mathrm{~Hz}), \beta(18-26 \mathrm{~Hz}), \gamma(35-45 \mathrm{~Hz})$, and high- $(80$ $100+\mathrm{Hz}$ ) frequency bands. The frequency bands power changes magnitude along with real or imagined movements $[6,7]$. A screening task identifies electrodes that reveal signal amplitude changes when responding to behaviour changes. Subjects are shown visual cues on a monitor that prompts them to imagine a movement or sound.

There are many methods for ECoG signals classification which differ in features and classifiers. Used features are based on extracted features, feature reduction methods and feature selection measures. Samiee et al., [8] used different algorithms with different results for a specific data set. The need to find the best algorithm to do a five-class finger flexion classification is to choose flexed finger among one hand's fingers. To achieve this, after feature extraction, different feature reduction methods and classification examined training data and chose the best algorithm according to the results. Niederhofer et al., [9] obtaining new results in a segmented electrode signals spatio temporal linear prediction using long-term SEEG and ECoG recordings of patients in epilepsy was discussed in detail.

A BCI system includes brain signals acquisition, processing and classification of acquired signals, feedback of interpreted brain state, and using classified signals to perform tasks. Improvements in BCI systems are possible by improving any subsystem. A BCI system that pre-processes and extracts features from ECoG signals using Wavelet Packet Tree and Common Spatial Pattern is presented. Features are normalized using $\mathrm{z}$ score normalization and are fused with Median Absolute Deviation. The feature level fusion of the feature vectors has better discrimination power than the individual vectors. It is observed that the proposed fusion significantly improves the performance of the classifiers. Fused features are classified with $\mathrm{k}$ Nearest Neighbor and Naïve Bayes. The rest of the paper is organized as follows: section 2 reviews 
some selected works available in the literature, section 3 presents the methodology, and the results in section 4 . Section 5 concludes the paper.

\section{RELATED WORKS}

A new algorithm to classify single-trial ECoG during motor imagery was proposed by Wei et al., [10]. Nonlinear regressive coefficients were extracted in 2 frequency bands of $0-3 \mathrm{~Hz}$ and $8-30 \mathrm{~Hz}$ as classification features. A Genetic Algorithm (GA) selected optimal feature subset and a Support Vector Machine (SVM) for evaluation. A 7\% generalization error was achieved on BCI Competition III's data set I.

A BCI using ECoG was proposed by Zhao et al., [11]. Feature extraction is a task that affects classification results. Discrete wavelet transform was first applied to ECoG signals from one subject performing imagined movements of either left smallfinger or tongue. After preprocessing, relative wavelet energy of selected 8 channels was extracted and a 40 dimension feature vector built. Then feature vector dimension $\mathrm{f}$ was reduced using Principal Component Analysis (PCA). Finally, Probabilistic Neural Network (PNN) classified it. 91.8\% maximum average classification accuracy was reached when the spread of radial basis functions was 0.11 . Offline analysis revealed that ECoG signals could be used in BCI, and suggested new ideas for feature extraction and imaginary movement's classification in ECoG-based BCI research.

A SVM ensemble method for ECoG identification to solve low classification accuracy problems and weak robustness for ECoG collection during different times was proposed by Li et al., [12]. Common Spatial Pattern (CSP) algorithm extracted features and SVM ensemble classified ECoG. Also, bagging algorithm and cross-validation technique were used in individual SVM Ensemble generation. The results verified that SVM ensemble accuracy was better than that of single SVM for ECoG collection at different times, and crossvalidated technique had better performance than bagging. So, SVM ensemble has stronger robustness and generalization ability compared to individual SVMs, and improved ECoG signals classification.

Automatic classification of ECoG signals into normal, interictal and ictal periods which occur during epileptiform activity for epilepsy diagnosis was performed by Kutlu and Kose [13]. For feature extraction, discrete Fourier transform and Hjorth descriptors were used and k-Nearest Neighbor (kNN) and Multilayer Artificial Neural Networks (MLANN) for classification. Results showed that the new feature extraction method was superior performance when used with MLANN for classification. The recognition rates obtained were $97.45 \%$ and $99.75 \%$ for training-test sets selection from different and same channels, respectively.

Feature extraction using band powers was applied to ECoG signals from one subject performing imagined movements of either left small-finger or tongue as proposed by Zhao et al., [14]. PNN suitable for classification problems classified two different imaginary movements. Classification accuracy rate for test data set reached a maximum of $86 \%$ when radial basis functions spread was 0.38 . The results showed that ECoG signals were usable and proved powerful in BCI system design.

The problem of classifying multichannel ECoG related to individual finger movements for BCI was tackled by Onaran et al., [15]. For this it applied a recently developed hierarchical spatial projection neural activity framework for ECoG feature extraction. The algorithm extended binary common spatial patterns algorithm to multiclass problem constructing a redundant spatial projections set tuned for finger movements paired and group-wise discrimination. Groupings were constructed by merging data of adjacent fingers and contrasting them with the rest, like the first two fingers (thumb and index) vs. others (middle, ring and little). It applied this to BCI competition IV ECoG data recorded from 3 subjects. It observed that maximum classification accuracy was from gamma frequency band $(65-200 \mathrm{~Hz})$. For this frequency range, average classification accuracy over 3 subjects was $86.3 \%$ indicating that redundant spatial projection framework can successfully decode finger movements for BMI from ECoG.

A new algorithm to classify single-trial ECoG during motor imagery was proposed by Wei and Tu [16]. Optimal channel subsets were selected by GAs from multi-channel ECoG recordings, then power features were extracted through CSP and finally Fisher discriminant analysis (FDA) classified it. The algorithm was applied to BCI Competition III's Data set I and $90 \%$ classification accuracy was achieved on test set using seven channels.

The Elghrabawy and Wahed [17] study was a step to explore ECoG signals usability as a BCI input technique and multidimensional BCI control. Signal processing/ classification were validated to predict kinematic parameters for 5-class finger flexion. Signal was provided by BCI competition IV ECoG dataset. Shift invariant wavelet decomposition and multi-taper frequency spectrum were used for feature extraction. Multilayer perceptron and pace regression classified it. Results revealed that predicted finger movement correlated with movement states.

Using a classifier to differentiate preictal from interictal ECoG signals led to the proposal of a patient-specific seizure prediction algorithm. ECoG spectral power processed in 4 different fashions were features: time-differential, raw, spacedifferential, and time/space-differential ECoG. Features were classified with cost-sensitive SVMs by double crossvalidation methodology. Park et al., [18] proposed an algorithm which was applied to 18 patients ECoG recordings in a Freiburg EEG database, totaling 80 seizures and 437hour-long interictal recordings. Classification with feature from time/space-differential ECoG revealed $86.25 \%$ sensitivity and 0.1281 false positives per hour in out-ofsample testing.

An algorithm to classify motor imagery ECoG signals, recorded in various sessions was proposed by Aydemir and Kayikcioglu [19]. kNN method classified extracted feature vectors with wavelet transform. The new algorithm was successfully applied to BCI competition 2005's Data Set I achieving $95 \%$ classification accuracy on test set.

A new approach to analyze ECoG trails in session-to-session transfer exits was proposed by Yuan et al., [20] where first dimension reduction was performed with ICA decomposition. Next, ECoG trials were clustered through an unsupervised learning algorithm called affinity propagation. Primary results reveal that the new approach ensured reasonable results compared to using classical K-means clustering algorithm.

Algorithm for seizure onset and offset detection in rats' ECoG was presented by Park et al., [21]. As seizure in rats' ECoG evolves more stereotypically than in humans, analyzing seizure evolution in rats' ECoG helps understand evolution process. The new algorithm outperformed a prior automatic seizure detection/termination system in in-vivo rats' ECoG. It 
improves the algorithm using relevant $14-22 \mathrm{~Hz}$ to onsets and $7-45 \mathrm{~Hz}$ to offsets frequency bands; by using spectral power instead of spectral amplitudes for its feature; and replacing post processing 2-point moving-average filter with a 2nd order Kalman filter. Not only does the new algorithm ensure better detection statistics, it also lowers system's complexity by not requiring a fast Fourier transform computation and using a single structure with 2 different spectral power features for onset/offset detection.

An important neuroscience data analysis problem is estimating neural recordings dynamic biological and behavioral signals. An adaptive filtering paradigm to estimate dynamic state processes from mixed observation processes with point process and continuous valued observations was developed by Eden and Brown [22]. Analyzing filtering algorithms, it drew analogies to well-studied linear estimation algorithms like Kalman and Extended Kalman filters. It proved application of mixed filtering paradigm for recorded motor cortical spiking and Local Field Potential (LFP) activity. It showed that the mixed filter was able to capture movement trajectory information better than filters based on spiking activity or LFPs.

A nonlinear adaptive filter, Kernel Least Mean Square (KLMS) that nonlinearly maps inputs to higher dimensional feature space where inner products are computed for prediction of 2 dimensional hand trajectories from cortical surface recordings was used by Gunduz et al., [23]. The method was simple and effective nonlinear extension of Least Mean Square (LMS) algorithm. Preliminary results revealed great improvement in mean squared error (MSE) values of reconstructed trajectories compared to linear methods (LMS) at a 95\%confidence level in the highest excursion axis.

A new method for EEG classification in a BCI by adapting subject specific features in spectral, temporal and spatial domains was presented by Goksu et al., [24]. It extended earlier work on ECoG classification for this purpose, based on structural feature dictionary and used it to extract multichannel EEG recordings spectro-temporal patterns related to a motor imagery task. Feature dictionary construction was based on undecimated wavelet packet transform and extended to block FFT. It evaluated many subset selection algorithms to choose limited features for final classification. The new approach was tested on BCI Competition 2005 dataset- Iva's five subjects. By adapting wavelet filter for every subject, the algorithm achieved an average $91.4 \%$ classification accuracy. Classification results and selected features characteristics indicated that the new algorithm could jointly adapt to EEG patterns in spectrumspatio-temporal domain providing classification accuracies as good as current methods in literature.

\section{MATERIALS AND METHODS}

\subsection{Dataset}

Data Set I for BCI Competition III evaluated the new method having motor imagery in ECoG recordings. A subject must perform imagined movements of left small finger or tongue in a BCI experiment. The subject suffered from epilepsy and was not a locked-In patient. Hence, his neural activity was monitored for many days with ECoG recordings. These trials picked up electrical brain activity's time series. All recordings had a $1000 \mathrm{~Hz}$ sampling rate. Recorded potentials after amplification were stored as microvolt values. Each trial had an imagined tongue/imagined finger movement which was recorded for 3 seconds. To prevent data reflecting visually evoked potentials, recording intervals began 0.5 seconds after visual round ended. A 278 trial Brain activity was considered training data and 100 trials similar activity was considered test data [25].

Data sets consist of 278 trials performed in the first session (training data) and 100 trials from second session (test data). Electrical brain activity was picked up with an $8 \times 8 \mathrm{ECoG}$ platinum electrode grid placed on contralateral (right) motor cortex and covered by meninges and scull and was insensitive to muscle artifacts. As scull and meninges act as low-pass filters in EEG recordings, ECoG data contains stronger high frequency components than EEG. The grid covered right motor cortex completely, but due to its size (approx. $8 \times 8 \mathrm{~cm}$ ) it also recorded activity from nearby cortical areas. All recordings were performed with a $1000 \mathrm{~Hz}$ sampling rate. Recorded potentials after amplification were stored as microvolt values.

\subsection{Wavelet Packet Tree (WPT)}

Wavelet packets [26] represent a wavelet decomposition generalization and in the wavelet case decomposition is applied recursively to coarse scale approximations only (leading to pyramidal wavelet decomposition tree, see figure 1(a)). But, in Wavelet Packet (WP) decomposition, recursive procedure is applicable to all, coarse scale approximations and detail signals (see figure 1(b)).

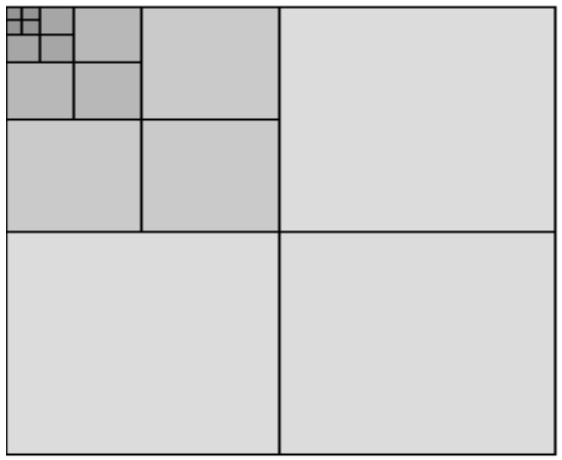

(a) Standard wavelet decomposition

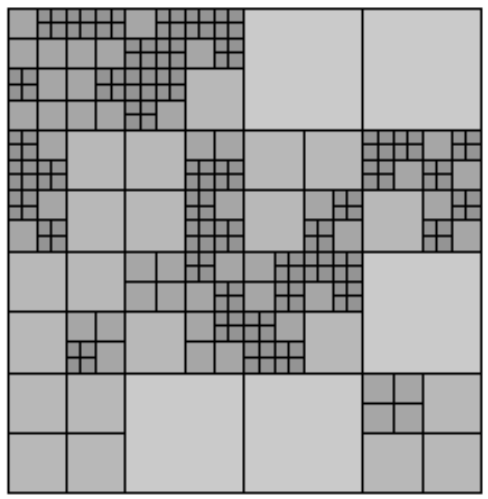

(b) Typical wavelet packet decomposition

Fig. 1 Decomposition trees for different subband structures

Wavelet Packet Tree (WPT) [27] is wavelets transform generalization providing good spectral and temporal resolutions in the time-frequency plane's arbitrary regions. Because of the uncertainty principle we know the area of a time frequency tile is constant: one cannot have both arbitrarily fine time and frequency resolutions. But, one can 
be traded for the other, looking for different tiling for every specific problem application (till they are produced by binary tree structures).

\subsection{Common Spatial Patterns (CSP)}

A feature extraction method, CSP algorithm learns spatial filters maximizing two classes discriminability. CSP is a popular algorithm for BCI design, during $\mathrm{BCI}$ competitions. CSP learns spatial filters which maximize band-pass filtered EEG signals variance from one class while minimizing variance from the other. As EEG signals variance filtered in a given frequency band correspond to signal power in this band, CSP achieves optimal BCI discrimination based on band power features. Formally, CSP uses spatial filters w, which extremizes following functions:

$$
j(W)=\frac{W^{T} X^{T} X_{2} W}{W^{T} X^{T} X_{2} W}=\frac{W^{T} C_{2} W}{W^{T} C_{2} W}
$$

Where $\mathrm{T}$ denotes transpose, $X_{i}$ is data matrix for class $\mathrm{i}$ (with training samples as rows and channels as columns) and $C_{i}$ is spatial covariance matrix from class $i$, assuming a zero mean for EEG signals. When EEG signals are band-pass filtered this last assumption is met. This optimization problem is solved (this is not the only way) by observing that function $\mathrm{J}(\mathrm{w})$ remains unchanged when filter $w$ is rescaled. Indeed $J(k w)=$ $\mathrm{J}(\mathrm{w})$, with $\mathrm{k}$ a real constant, meaning that rescaling of $\mathrm{w}$ is arbitrary. As such, extremizing $\mathrm{J}(\mathrm{w})$ is equivalent to extremizing $W^{T} \quad C_{l} w$ subject to constraint $W^{T} \quad C_{2} w=1$ as it is possible to rescale of $\mathrm{w}$ so that $W^{T} \quad C_{2} w=1$. Using Lagrange multiplier method, this constrained optimization problem extremizes the following function:

$$
L(\lambda, W)=W^{T} C_{1} W-\lambda\left(W^{T} C_{2} W-1\right)
$$

The filters $w$ extremizing $\mathrm{L}$ are such that the derivative of $\mathrm{L}$ with respect to w equals 0

$$
\begin{aligned}
& \frac{\partial L}{\partial W}=2 W^{T} C_{1} W-2 \lambda W^{T} C_{2} W \\
& C_{1} W=\lambda C_{2} W \\
& C_{2}^{-1} C_{1} W=\lambda W
\end{aligned}
$$

When using CSP, extracted features are the EEG signal variance logarithm after projection onto filters $\mathrm{w}$ [28].

Features are normalized using $\mathrm{z}$ score normalization and fused with Median Absolute Deviation. Attributes are normalized by scaling values so that they fall in a small-specified range, like 0.0 to 1.0. Normalization is useful for classification algorithms. There are many data normalization methods including $\mathrm{z}$-score normalization, min-max normalization and decimal scaling normalization.

Values for and attribute A are normalized based on mean and standard deviation of $\mathrm{A}$ in $\mathrm{z}$-score normalization. Value $\mathrm{v}$ of A is normalized to v' by computing:

$v^{\prime}\left((v-A) / \sigma_{A}\right)$

Where $\mathrm{A}$ and $\sigma_{\mathrm{A}}$ are mean and standard deviation of attribute A. This normalization method is useful when actual minimum/maximum of attribute A are unknown. The Median Absolute Deviation (MAD) is used to quantify variation. The median of all values is computed and difference of each value from median values is calculated in MAD. The difference between value and median is expressed as positive value and MAD is the median of that differences set.

\section{RESULTS AND DISCUSSION}

Experiments were conducted with BCI Competition III's Data Set I; 278 numbers of instances from the dataset were used. Features were extracted using Wavelet Packet Tree and proposed feature fusion. The instances were classified using 193 attributes. All the experiments were conducted for 10-fold cross validation. The classification accuracy and the Root Mean Square Error (RMSE) achieved are shown in the following figures.

Table 1. Classification Accuracy

\begin{tabular}{|l|l|l|}
\hline & \multicolumn{1}{|c|}{$\begin{array}{c}\text { Wavelet Packet } \\
\text { Tree }\end{array}$} & $\begin{array}{c}\text { Fusion } \\
\text { technique }\end{array}$ \\
\hline Naïve Bayes & 88.49 & 90.29 \\
\hline K NN & 88.21 & 89.57 \\
\hline
\end{tabular}



Figure 2: Classification Accuracy

It is observed from the experimental results that the feature fusion improves classification accuracy by $1.54 \%$ to $2.03 \%$. Naïve Bayes with feature fusion achieves the maximum accuracy of $90.29 \%$.

Table 2. RMSE

\begin{tabular}{|l|l|l|}
\hline & $\begin{array}{c}\text { Wavelet Packet } \\
\text { Tree }\end{array}$ & $\begin{array}{c}\text { Fusion } \\
\text { technique }\end{array}$ \\
\hline Naïve Bayes & 0.2682 & 0.2177 \\
\hline K NN & 0.2714 & 0.2215 \\
\hline
\end{tabular}

The RMSE reduces significantly when the classifiers use fused features for classification. It is observed that the RMSE reduces by $20.79 \%$ for Naïve Bayes and by 20.25 for kNN when fused features are used. 


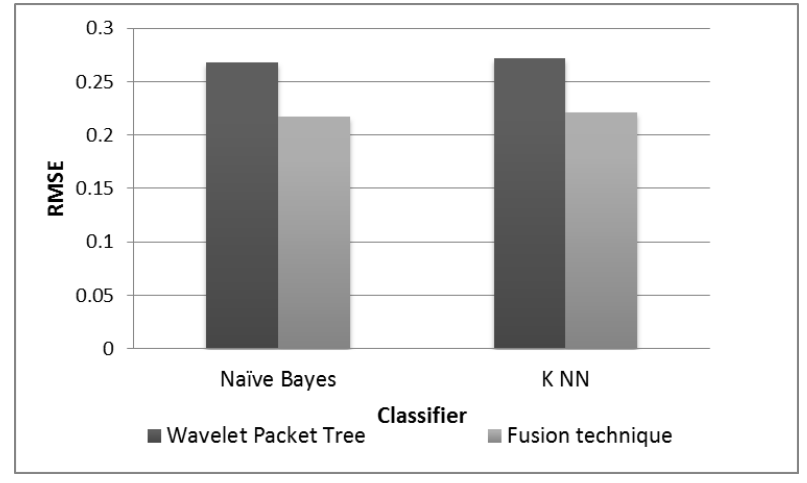

Figure 3: RMSE

Table 3. Recall

\begin{tabular}{|l|l|l|}
\hline & \multicolumn{1}{|c|}{$\begin{array}{c}\text { Wavelet Packet } \\
\text { Tree }\end{array}$} & $\begin{array}{c}\text { Fusion } \\
\text { technique }\end{array}$ \\
\hline Naïve Bayes & 0.8849 & 0.9029 \\
\hline K NN & 0.8821 & 0.8957 \\
\hline
\end{tabular}

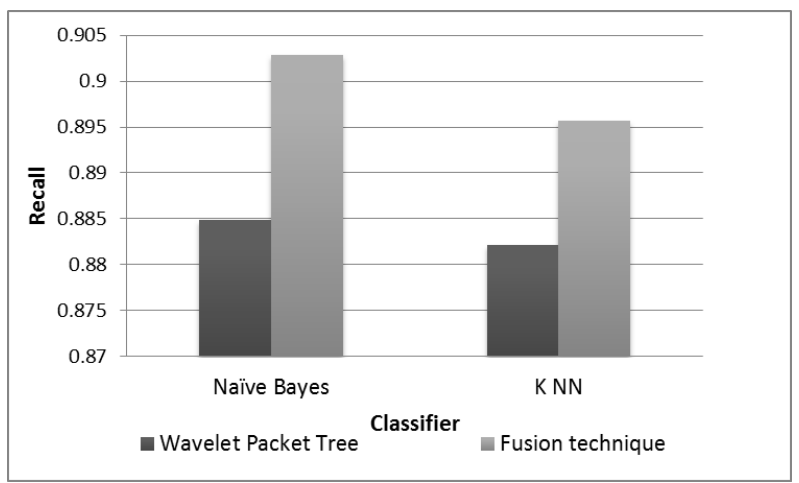

Figure 4: Recall

It is observed from the experimental results that the feature fusion improves recall of the classifiers by $1.53 \%$ to $2.01 \%$. Naïve Bayes with feature fusion achieves the maximum recall of 0.9029 .

Table 4. Precision

\begin{tabular}{|l|l|l|}
\hline & \multicolumn{1}{|c|}{$\begin{array}{c}\text { Wavelet Packet } \\
\text { Tree }\end{array}$} & $\begin{array}{c}\text { Fusion } \\
\text { technique }\end{array}$ \\
\hline Naïve Bayes & 0.8862 & 0.9031 \\
\hline K NN & 0.8822 & 0.8962 \\
\hline
\end{tabular}

The precision improves significantly when the fused features are used for classification. It is observed that the precision increases by $1.89 \%$ for Naïve Bayes and by $1.57 \%$ for $\mathrm{kNN}$ when fused features are used.

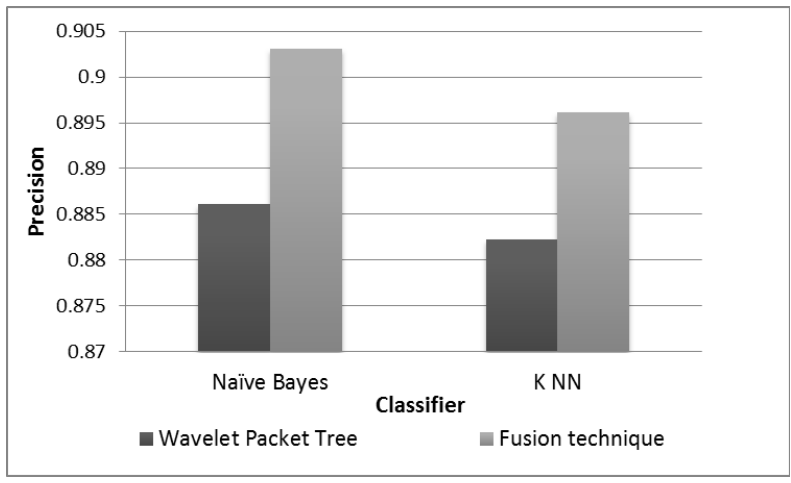

Figure 5: Precision

\section{CONCLUSION}

$\mathrm{BCI}$ is a clinical application that might restore communication to those suffering from severe motor disabilities. Electrophysiological brain signals recording and analysis is the base of BCI research. ECoG is an invasive record to brain signals from electrode grids on the brain's surface. ECoG signal ensures localization of neural signals source regarding brain functions due to its high spatial resolution. Feature extraction is a task that affects classification results. This paper presents a BCI system pre-processing and extracting features from ECoG signals using Wavelet Packet Tree and Common Spatial Pattern. Features are normalized using $\mathrm{z}$ score normalization and fused using Median Absolute Deviation. BCI Competition III's Data Set I having motor imagery in ECoG recordings is used in the investigation. Results show that the new method ensured improved performance.

\section{REFERENCES}

[1] Wolpaw J. R. Brain-computer interface technology: a review of the first international meeting, Rehabilitation Engineering, IEEE Transactions on Neural Systems and Rehabilitation, Vol. 8, Issue. 2, 2000, pp. 164-173.

[2] Kaur, M., Ahmed, P., \& Qasim Rafiq, M. (2012). Technology Development for Unblessed People using BCI: A Survey. International Journal of Computer Applications, 40.

[3] Wilson, J. A., Felton, E. A., Garell, P. C., Schalk, G., \& Williams, J. C. (2006). ECoG factors underlying multimodal control of a brain-computer interface. Neural Systems and Rehabilitation Engineering, IEEE Transactions on, 14(2), 246-250.

[4] Ball, T., Kern, M., Mutschler, I., Aertsen, A., and Schulze-Bonhage, A. (2009). Signal quality of simultaneously recorded invasive and non-invasive EEG. Neuroimage 46, 708-716.

[5] Brunner, P., Ritaccio, A. L., Lynch, T. M., Emrich, J. F., Wilson, J. A., Williams, J. C., Aarnoutse, E. J., Ramsey, N. F., Leuthardt, E. C., Bischof, H., and Schalk, G. (2009). A practical procedure for real-time functional mapping of eloquent cortex using electrocorticographic signals in humans. Epilepsy Behav. 15, 278-286.

[6] B. Graimann, J. E. Huggins, A. Schlogl, S. P. Levine, and G. Pfurtscheller, "Detection of movement-related desynchronization patterns in ongoing single-channel electrocorticogram," IEEE Trans. Neural Syst. Rehabil. Eng., vol. 11, no. 3, pp. 276-281, Sep. 2003. 
[7] E. C. Leuthardt, G. Schalk, J. R. Wolpaw, J. G. Ojemann, and D. W. Moran, "A brain-computer interface using electrocorticographic signals in humans," J. Neural Eng., vol. 1, pp. 63-71, 2004.

[8] Niederhofer, C., Gollas, F., \& Tetzlaff, R. (2007, August). Dynamics of EEG-signals in epilepsy: Spatio temporal analysis by Cellular Nonlinear Networks. InCircuit Theory and Design, 2007. ECCTD 2007. 18th European Conference on(pp. 296-299). IEEE.

[9] Samiee, S., Hajipour, S., \& Shamsollahi, M. B. (2010, June). Five-class finger flexion classification using ECoG signals. In Intelligent and Advanced Systems (ICIAS), 2010 International Conference on (pp. 1-4). IEEE.

[10] Wei, Q., Gao, X., \& Gao, S. (2006, August). Feature extraction and subset selection for classifying single-trial ECoG during motor imagery. In Engineering in Medicine and Biology Society, 2006. EMBS'06. 28th Annual International Conference of the IEEE (pp. 1589-1592). IEEE.

[11] Zhao, H. B., Yu, C. Y., Liu, C., \& Wang, H. (2010, October). ECoG-based brain-computer interface using relative wavelet energy and probabilistic neural network. In Biomedical Engineering and Informatics (BMEI), 2010 3rd International Conference on (Vol. 2, pp. 873877). IEEE.

[12] Li, M., Yang, J., Hao, D., \& Jia, S. (2009, December). ECoG recognition of motor imagery based on SVM ensemble. In Robotics and Biomimetics (ROBIO), 2009 IEEE International Conference on (pp. 1967-1972). IEEE.

[13] Kutlu, F., \& Kose, C. (2013, April). Epileptic seizure detection from ECoG signals acquired with experimental epilepsy. In Signal Processing and Communications Applications Conference (SIU), 2013 21st (pp. 1-4). IEEE.

[14] Zhao, H. B., Liu, C., Wang, H., \& Li, C. S. (2010, August). Classifying ECoG Signals Using Probabilistic Neural Network. In Information Engineering (ICIE), 2010 WASE International Conference on (Vol. 1, pp. 7780). IEEE.

[15] Onaran, I., Ince, N. F., \& Cetin, A. E. (2011, August). Classification of multichannel ECoG related to individual finger movements with redundant spatial projections. In Engineering in Medicine and Biology Society, EMBC, 2011 Annual International Conference of the IEEE (pp. 5424-5427). IEEE.

[16] Wei, Q., \& Tu, W. (2008, August). Channel selection by genetic algorithms for classifying single-trial ECoG during motor imagery. In Engineering in Medicine and Biology Society, 2008. EMBS 2008. 30th Annual International Conference of the IEEE (pp. 624-627). IEEE.

[17] Elghrabawy, A., \& Wahed, M. A. (2012, December). Prediction of five-class finger flexion using ECoG signals. In Biomedical Engineering Conference (CIBEC), 2012 Cairo International (pp. 1-5). IEEE.

[18] Park, Y., Netoff, T., \& Parhi, K. (2010, March). Seizure prediction with spectral power of time/space-differential EEG signals using cost-sensitive support vector machine. In Acoustics Speech and Signal Processing (ICASSP), 2010 IEEE International Conference on (pp. 5450-5453). IEEE.

[19] Aydemir, O., \& Kayikcioglu, T. (2010, April). Motor imagery ECoG signals classification using wavelet transform features. In Signal Processing and Communications Applications Conference (SIU), 2010 IEEE 18th (pp. 296-299). IEEE.

[20] Yuan, Y., Xu, A. B., Guo, P., \& Zhang, J. C. (2008, October). ECoG analysis with affinity propagation algorithm. In Natural Computation, 2008. ICNC'08 Fourth International Conference on (Vol. 5, pp. 52-56). IEEE.

[21] Park, Y. S., Netoff, T. I., Yang, X., \& Parhi, K. K. (2012, August). Seizure detection on/off system using rats' ECoG. In Engineering in Medicine and Biology Society (EMBC), 2012 Annual International Conference of the IEEE (pp. 4688-4691). IEEE.

[22] Eden, U. T., \& Brown, E. N. (2008, March). Mixed observation filtering for neural data. In Acoustics, Speech and Signal Processing, 2008. ICASSP 2008. IEEE International Conference on (pp. 5201-5203). IEEE.

[23] Gunduz, A.; Jung-Phil Kwon; Sanchez, J.C.; Principe, J.C., (2009) "Decoding hand trajectories from ECoG recordings via kernel least-mean-square algorithm," Neural Engineering, 2009. NER '09. 4th International IEEE/EMBS Conference on , vol., no., pp.267,270.

[24] Goksu, F., Ince, N. F., Tadipatri, V. A., \& Tewfik, A. H. (2008, August). Classification of EEG with structural feature dictionaries in a brain computer interface. In Engineering in Medicine and Biology Society, 2008. EMBS 2008. 30th Annual International Conference of the IEEE (pp. 1001-1004). IEEE.

[25] Thomas Lal, Thilo Hinterberger, Guido Widman, Michael Schröder, Jeremy Hill, Wolfgang Rosenstiel, Christian Elger, Bernhard Schölkopf, Niels Birbaumer. Methods Towards Invasive Human Brain Computer Interfaces. Advances in Neural Information Processing Systems

[26] M.Wickerhauser. Adapted wavelet analysis from theory to software. A.K. Peters, Wellesley, Mass., 1994.

[27] R.R. Coifman and M.V. Wickerhauser, "Entropy-Based Algorithms for Best Basis Selection," IEEE Trans. Inform. Theory, vol. 38, 1992, pp. 1713-1716.

[28] Lotte, F., \& Guan, C. (2011). Regularizing common spatial patterns to improve BCI designs: unified theory and new algorithms. Biomedical Engineering, IEEE Transactions on, 58(2), 355-362. 